\title{
Mathematical models of plant metabolism
}

Hai Shi, Jörg Schwender

Address:

Biological, Environmental and Climate Sciences Department

Brookhaven National Laboratory

Upton, New York 11973

Tel: (631) 344-3797

E-mail: Schwend@bnl.gov

Corresponding author: Jörg Schwender 


\begin{abstract}
Among various modeling approaches in plant metabolic research, applications of constraintbased modeling are fast increasing in recent years, apparently driven by current advances in genomics and genome sequencing. Constraint-Based modeling, the functional analysis of metabolic networks at the whole cell or genome scale, is more difficult to apply to plants than to microbes. Here we discuss recent developments in Constraint-Based modeling in plants with focus on issues of model reconstruction and flux prediction. Another topic is the emerging application of integration of Constraint Based modeling with omics data to increase predictive power. Furthermore, advances in experimental measurements of cellular fluxes by ${ }^{13} \mathrm{C}$ Metabolic Flux Analysis are highlighted, including instationary ${ }^{13} \mathrm{C}-\mathrm{MFA}$ used to probe autotrophic metabolism in photosynthetic tissue in the light.
\end{abstract}

\title{
Abbreviations
}

CBM, constraint-based modeling; FBA, flux balance analysis; FVA, flux variability analysis, ${ }^{13} \mathrm{C}$ MFA, ${ }^{13} \mathrm{C}$-metabolic flux analysis 


\section{Introduction}

Metabolic processes in a living cell can be understood as resulting from the activity of a highly complex network of biochemical reactions. Attempts to rational re-design metabolism often result in unexpected or undesirable results [1]. Mathematical models have proven to be a useful guide for metabolic engineering, in particular to optimize the production of various useful chemicals in microorganisms [2]. A number of useful and valuable biochemicals can be obtained directly from plants in a sustainable way [3]. The virtue of mathematical modeling for metabolic engineering and systems biology is gaining attention in the plant science community [3]. In this review recent advances in Constraint-Based Modeling (CBM) for functional analysis of metabolic networks and ${ }^{13} \mathrm{C}$-Metabolic Flux Analysis $\left({ }^{13} \mathrm{C}-\mathrm{MFA}\right)$ for empirical flux determination in plants are highlighted, as well as their synergies with data obtained from transcriptomics, metabolomics, and ${ }^{13} \mathrm{C}-\mathrm{MFA}$.

\section{Constraint-based modeling in plants}

Constraint-based modeling (CBM) of metabolism is commonly understood as a systems biology methodology that allows to bridge the gap between a genome encoded parts list and phenotypic whole-cell flux states. Core concepts of CBM, as defined recently by Lewis et al. [4], are summarized here in brief: 1) Relying on genomic and biochemical information, whole-cell metabolic networks are defined based on reaction stoichiometry. 2) Typically there are a large number of computable metabolic flux phenotypes ('solution space'). Physiologically non-relevant ones can be excluded by addition of physiological or physicochemical constraint equations to the model. 3) Often relevant flux states are selected by linear optimization (Flux Balance Analysis, FBA), based on an assumed cellular objective. Following the publication of the first reconstruction of Haemophilus influenzae metabolism based on a completely sequenced genome in 1999 [5] the number of published microbial models has been ever-growing and is rapidly increasing during recent years [6]. A variety of computational tools for model reconstruction and $\mathrm{CBM}$ is now available, allowing global and unbiased in-silico exploration of metabolism in various ways, like e.g. the prediction of cellular flux states, prediction of mutant phenotypes or devising strategies for metabolic engineering $[4,6,7,8]$. After the rise of microbial CBM, the number of plant related CBM studies begins to rise as well [9-34]. Since higher plants are multicellular organisms, CB modeling is often being extended to simulate interaction between different cell types [15*,32], to simulate metabolic / physiological gradients in tissues $\left[11^{*}\right]$, or to simulate primary $\mathrm{CO}_{2}$ assimilation and aspects of the diurnal metabolic changes on a 
whole plant scale $\left[16^{*}\right]$. Another study inferred possible evolutionary intermediates between $\mathrm{C}_{3}$ and $\mathrm{C}_{4}$ types of photosynthesis based on integrating $\mathrm{CB}$ models with mechanistic models of photosynthesis [21*]. In addition, integration of CB models of leaf, stem and seed cells into a whole plant / multi-scale model framework was reported, exploring carbon partitioning during development of barley plants $\left[18^{*}\right]$.

With the number of complete genome sequences for higher plants and crop plants now rapidly increasing [35, 36], a development similar to the explosion of microbial CBM might be possible. However, the current potential of the modeling approach to explore the metabolic potential of plant species has to be evaluated considering the still underexplored complexity of plant metabolism. Genome-scale metabolic models are commonly referred to as metabolic reconstructions, which implicates that the entire metabolic potential encoded in a genome is represented in a model. The degree of perfection by which this can be achieved for a higher plant depends foremost on the completeness and accuracy of the functional annotation of a genome. To obtain a sense for the current quality of functional annotation, one might compare Escherichia coli and Arabidopsis thaliana [37] as the best-studied model organisms in the microbial and the plant world, respectively. Currently the number of publications related to Escherichia coli found in the life-sciences bibliographic database MEDLINE is about 50 times the number of genes in the genome. In case of Arabidopsis thaliana, this quantity, known as the Species Knowledge Index [38], is only about one, suggesting that this most studied plant model species might still be considered vastly underexplored. $E$. coli $\mathrm{CB}$ models now have been under development and refinement for about 15 years [8]. For the E. coli reconstruction iJO1366, 97\% of the 1366 genes in the model have annotation of metabolic function based on experimental evidence [39]. In difference, it was reported that only $43 \%$ of enzymes described in the $A$. thaliana pathway database AraCyc have experimental support to some degree [40]. Therefore, even for the best-studied model plant species the currently available genome-scale metabolic networks are largely defined based on computational prediction of gene function. For all other plant genomes sequenced to date gene function can be considered to be defined almost exclusively based on computational prediction [41]. In essence the various existing methods of computational prediction of gene function rely on the presence of a set of experimental proven gold standards [41] and it has been argued that the goal of understanding the function of all genes in a plant genome can only be reached if the current gold standard set is significantly expanded, in particular by characterization of genes in other plants than $A$. thaliana [41]. Therefore, while there has been much effort in improving comparative genomics approaches 
and to develop automated and streamlined workflows for genome annotation and network reconstruction [42-44], development of high throughput experimental gene discovery and enzyme characterization $[45,46]$ will be crucial to advance plant genomics and CBM in plants. From three recent $A$. thaliana genome scale reconstructions $\left[23,28,30^{*}\right]$ we could extract a total of 2740 unique gene locus ID's. Chae et al. [44] computationally predicted 6746 enzymes in Arabidopsis, associated to 5265 gene loci. This means that current $A$. thaliana genome scale models might represent just about $50 \%$ of all genes encoding for metabolic functions. In addition to functional annotation, the knowledge on subcellular localization of enzymes is limited in plants. The currently probably most comprehensive database collecting information on subcellular localization of proteins in plants is SUBA3 [47]. The database contains published experimental data and computational predictions on protein location in Arabidopsis for 11 subcellular compartments [47]. While the various localization data for a specific protein are often contradictory, a naïve Bayes approach was developed to integrate the data to provide a single location call for proteins [48].

Another challenge for the CBM approach is the prevalence of enzyme promiscuity. In plant secondary metabolism, enzymes with relaxed substrate and reaction specificity (also "generalist enzymes",[49]) seem to be more the rule than exception [50,51]. The extent of enzyme promiscuity and its relevance for CBM has been assessed recently for $E$. coli [52]. For example, $37 \%$ of the E. coli enzymes were found to be generalist enzymes. Specialist enzymes (catalyzing one reaction on a preferred substrate) tend to be essential and maintain higher flux than generalist enzymes [52]. In plants, while primary metabolism tends to be evolutionary conserved, secondary metabolism is evolutionary fast expanding and highly diversified among land plants [44], i.e. it likely represents much of what distinguishes different land plant species, but at the same time is hard to define in CB models.

In similar to enzyme catalyzed reactions, transport between sub-cellular compartments and inbetween cells and tissues is a significant part of what defines plant physiology and metabolism $[53,54]$. At the molecular level metabolic transport is considered to be vastly underexplored $[55,56]$. For $A$. thaliana, protein structure prediction identified 1800 genes that could encode membrane proteins with transport functions [57]. In CBM, the lack of evidence on intracellular transport means that putative transmembrane transports have to be added to a network as needed to achieve overall pathway functionality [58]. In difference to such hypothetical single metabolite transports, many of the known trans-membrane transport mechanisms, in particular across mitochondrial and chloroplast membranes, facilitate strict and specific sym- or antiport of metabolites that are substrates of the same enzyme reaction or pathway $[55,59]$. Such 
stoichiometric coupling via transmembrane flux is integral to highly compartmentalized processes like photosynthesis, photorespiration, and others [60] and might be defined as a hallmark feature of intracellular metabolic crosstalk.

In light of the discussed challenges to the definition of plant CB models on a genome scale, the development and application of bottom-up metabolic reconstructions is noteworthy $\left[1,9^{*}, 17,19^{*}\right.$, 22,61-65]. In such a bottom-up approach emphasis is given on high quality manual curated pathway functionalities required for uptake of physiologically relevant substrates and for synthesis of biomass compounds most relevant in a specific tissue / cell type. Due to this focus these reconstructions tend to benefit more from mining of original literature and expert domain knowledge instead of reliance on information collected from databases. Arnold and Nikoloski $\left[9^{*}\right]$ compared simulations of autotrophic biomass synthesis between their core metabolism model of Arabidopsis leafs and several published genome-scale reconstructions. They concluded that their model is comparable to other genome-scale Arabidopsis models, in particular with regards to the ability to grow under different physiological conditions, efficiency of carbon utilization and flexibility in the use of alternative pathways [9*].

\section{Predicting flux states by optimization}

One of the most widely used methods applied with CBM is Flux Balance Analysis (FBA). Based on the network stoichiometry and within the given flux bounds (Figure 1A), the FBA procedure finds a cellular steady state flux distribution that meets a metabolic optimality criterion (objective function) (Figure 1B). Objectives commonly used for microbial CBM can be related to the idea that evolutionary pressures require an organism to grow with maximal possible biomass yield (often referred to as maximal growth rate), to make most efficient use of substrates, to produce cellular energy most efficiently (maximal ATP yield), or to distribute flux load to use enzyme capacity most efficiently [66]. However, it has been pointed out that assuming that the cellular flux distribution is governed by a single optimality criterion might not be realistic and that a specific criterion might only be justifiable for one organism under a specific physiological condition [66]. Later Schuetz et al. [67] proposed that in-vivo flux states in microbes evolve to be close to optimality, but also in a way that allows adjusting between physiological conditions by minimal flux adjustments. In plant related FBA studies $\left[11^{*}, 12,13^{*}, 16^{*}-19^{*}, 22,25-27,61,68\right]$, the cellular objectives are typically adopted from microbial studies. Working on heterotrophically growing Arabidopsis cell cultures, Cheung et al. [13*] benchmarked the use of different objective functions against experimental flux measurements $\left({ }^{13} \mathrm{C}-\mathrm{MFA}\right)$. They found that adjustments in 
model constraints defining cellular energy maintenance cost have more impact on the fidelity of flux predictions than the choice of the objective function $\left[13^{*}\right]$. In conclusion, flux solutions produced by optimization (FBA) should not necessarily be taken as in-vivo flux predictions. It has to be emphasized here that besides for the purpose of interpretation of cell internal flux states, FBA is integral to a number of other types of phenotype exploration not further discussed here [4].

In CBM it is often desirable to be able to pinpoint a specific flux state by FBA in order to interpret cell-internal flux solutions in a biological context. However, a general limitation of CBM is that the flux space most often cannot be uniquely resolved due to missing constraints $[69,70]$. While the standard FBA procedure will find one steady state flux solution to the optimization problem (Figure 1B), many alternative optima exist for most of the commonly used cellular objectives [71]. A number of plant studies therefore have made use of an extended FBA approach in this context $\left[12,13^{*}, 16-18,21^{*}, 22,25,26^{*}, 27,61,68\right]$. Two optimizations are applied in succession to narrow down the solution space to a single solution. A first optimization defines the exchanges across the system boundary (Figure 1B) while in a secondary optimization a minimum of total flux across the network is sought by use of a linear $\left[12,13^{*}, 16^{*}, 21^{*}, 25,26^{*}, 27\right]$ (Figure $1 \mathrm{C}$ ) or quadratic flux sum minimization $\left[17,18^{*}, 22,61,68\right]$ (Figure 1D). Flux sum minimization avoids loop artifacts as shown in Figure $1 \mathrm{~B}$ and has been rationalized based on the need of an organism to make efficient use of enzyme capacity $[66,72]$. However, it appears that flux sum minimizations in Figures $1 \mathrm{C}$ and 1D result in different internal solutions. The toy model illustrates that trying to decide on which of the two flux states is more realistic might not be meaningful. As the case in the toy model (Figure 1A), in real CB models there is no reliable information on flux capacity (flux bounds) for most if not all of the internal reactions. Also, like in the toy model, in plant metabolic networks redundant pathway functionalities are common and therefore precise predictions on cell-internal flux distribution, although computationally possible and convenient to interpret, might constitute over-stringency in optimization. If better information on the reaction bounds in the network could be obtained, e.g. by ${ }^{13} \mathrm{C}-\mathrm{MFA}$, the flux space can be narrowed down (Figure 1G). Otherwise, instead of seeking unique flux states by FBA, some recent plant studies used less stringent explorations of the solution space $\left[21^{\star}, 29^{*}, 33,34\right]$. Flux Variability Analysis (FVA) [71] determines the boundaries of the optimal solution space as numerical ranges (Figure 1E, F). Accordingly, recent plant studies extensively used FVA $\left[19^{*}, 29^{*}, 62\right]$. The optimum flux intervals determined by FVA can be interpreted by qualitative categorization [62]. For example, under optimality some reactions are bounded to zero, i.e. are 
never used in any particular flux solution and considered sub-optimal. If a reaction is bounded by an interval that does not include zero, simulation of the knockout of this reaction causes suboptimal flux states or even lethality (reaction essentiality). Quantitative interpretations of flux bounds have been discussed in a study on pathway usage in dependence on trade-off between different storage compounds in a developing seed [29*].

\section{Integration of genome-scale models with omics data}

Due to the general sparsity of empirical data to constrain the solution space of CB models, integration with omics data has been recognized as a means to improve predictive power $[70,73,74]$. Next generation sequencing typically provides comprehensive coverage of gene expression and is a mature and highly automated technology available at reasonable cost [70]. In principle, the flux through a reaction depends on enzyme abundance and hence on transcript level. However, there are several complex layers of cellular processes between gene expression and metabolic activity and literature provides numerous examples of discordance between transcript, protein, metabolite abundance and metabolic flux, in particular with regards to central metabolism in microorganisms [75] and in plants $\left[76^{\star}\right]$. In view of this substantial gap between measured transcript levels and flux, a portfolio of diverse methods for integration of transcriptomic data with CB models has been developed and been reviewed in detail elsewhere $[73,74]$. One class of these methods derives condition- or cell type specific CB models as a functional sub-network of the genome-defined organism-wide metabolic network. Accordingly, recent plant studies use transcript data to derive cell type specific [32] or organ specific metabolic networks [30]. A very recent development towards integration of expression data is an extension of $C B$ modeling, so-called modeling of metabolism and macromolecular expression (ME) [77]. ME models capture major aspects of macromolecular synthesis by incorporating the metabolic cost for gene expression in much detail. ME-models explicitly account for the investment into the synthesis of all enzymes necessary for operating a metabolic pathway. Therefore the tradeoff between alternative pathways is expected to become more realistic in ME simulations [77]. Future application of this principle to plant models might be possible, but this approach hasn't yet been reported for networks of the size of a eukaryotic organism.

Besides transcriptomics, metabolomics data have been integrated into CB models [33,34]. Here a basic challenge is that metabolite concentrations per se cannot be used as model constraint and cannot be predicted by CBM, since the mathematical formalism doesn't explicitly include 
metabolite concentrations. Yet, various mathematical derivatives can be obtained from CB models and used as a proxy to metabolite concentration or to condition specific differences in metabolite concentration [24,78-81]. For example, steady-state flux states derived by CBM can be transformed into a metabolite-centric perspective by summation of all incoming and outgoing fluxes around a particular metabolite [79]. This so-called flux-sum approach has been proposed as a proxy for metabolite concentration [79] or the metabolite turnover rate [80]. In plants, studying nitrogen metabolism in maize leaves, Simons et al. [32] applied the flux-sum method to predict perturbations of metabolism by different nitrogen nutrient status or by mutations. They compared condition-specific differences in measured metabolite levels to changes in the model predicted flux-sum. In particular when transcriptomic and proteomic data were included as additional constrains on the flux solution space, the flux-sum approach predicted the changes in the metabolome with good accuracy [32]. In another study on acclimation of Arabidopsis to varying light and temperature conditions [34], temporal variability in metabolite levels is related to changes in flux capacity of metabolic pathways, predicted by integration of transcriptomics data $[33,34]$.

Notably, in studies like the above discussed the subcellular distribution of metabolites among cytosol, plastids, mitochondria and other compartments is not resolved since metabolite profiles were obtained from tissue extraction. Using the technique of non-aqueous fractionation to separate organelles prior to extraction, Nägele and Weckwerth [24] partially resolved subcellular distribution of metabolites. The authors present a workflow to relate differences in metabolite levels, measured under different physiological conditions, to the structure of a compartmentalized Arabidopsis genome-scale network. In essence, two metabolic states can be compared and elasticities (sensitivity of reaction rates to changes of the metabolite concentrations) are numerically approximated. As a main predictive outcome the authors find that a major difference between cold sensitive and tolerant accessions of Arabidopsis exists in the ability to transport sucrose across the chloroplast envelope [24].

\section{Integration of flux measurements into CB model}

${ }^{13} \mathrm{C}$-Metabolic Flux Analysis $\left({ }^{13} \mathrm{C}-\mathrm{MFA}\right)$ is a well-established method to quantify intracellular fluxes and has been successfully applied in particular for plant cell- and tissue culture under heterotrophic or photo-mixotrophic growth conditions [82,83]. Flux maps of developing seeds have given insights into in-vivo contribution of alternative pathways to seed storage synthesis or energy cofactor balances [83]. Flux measurements are most directly linked to the CB modeling approach and informative (Figure $1 \mathrm{G}$ ). However, ${ }^{13} \mathrm{C}-\mathrm{MFA}$ does not have the potential broad 
applicability as given with transcriptomics, since ${ }^{13} \mathrm{C}-\mathrm{MFA}$ studies are typically dependent on well-defined cell culture conditions under quasi metabolic and isotopic steady state. Cheung et al. $\left[13^{*}\right]$ could show that the predictive fidelity of a CB model of Arabidopsis cell cultures is substantially improved if partial flux information derived from ${ }^{13} \mathrm{C}-\mathrm{MFA}$ is added as a constraint. In similar, Hay et al. [19*] probed central metabolism in cultured developing embryos of Brassica napus by ${ }^{13} \mathrm{C}-\mathrm{MFA}$. The flux distribution in central metabolism was transferred into a large scale CB model and the predictive potential of FVA substantially improved as judged by reduction of the flux solution space. Based on the ${ }^{13} \mathrm{C}-\mathrm{MFA}$ integration with CBM, highly resolved large-scale flux sets for two Brassica napus genotypes, differing in oil content, could be compared to differential expression derived in parallel experiments $\left[76^{\star}\right]$. With a few exceptions, differential activity in the major pathways of central metabolism (glycolysis, TCA cycle, amino acid, and fatty acid synthesis) was not reflected in contrasting abundances of the relevant transcripts. The conclusion was that transcript abundance on its own cannot be used to infer metabolic activity/fluxes in central plant metabolism [76*].

In two recent studies, metabolic flux analysis $\left({ }^{13} \mathrm{C}-\mathrm{MFA}\right)$ was integrated with other omics data to gain more insight into how regulation of metabolism and seed development takes place in developing oil seeds [84,85]. Li et al. [84] explored gene expression, metabolite and flux profiles of the soybean embryo in a time series during seed development. The data can be interactively mined. In a more targeted approach, Schwender et al. [85*] compared a set of 9 cultivars of oilseed rape with variation in lipid and starch content present in in-vitro culture. Flux, metabolite profiles and enzyme activities were measured along with proteomic data. Overall, among the cultivars a tradeoff between lipid and starch content was observed which was not reflected at the proteome level. Posttranslational metabolic control was indicated by numerous correlations between enzyme activity and flux as well as between levels of central metabolites and flux. Allosteric feedback control of glycolysis and starch synthesis appears to be involved in carbon partitioning between lipid and starch [85*].

\section{Autotrophic metabolism in plants requires isotopically non-stationary ${ }^{13} \mathrm{C}-\mathrm{MFA}$}

A typical approach of ${ }^{13} \mathrm{C}$-MFA is to trace label from ${ }^{13} \mathrm{C}$-labeled multi-carbon substrate through central metabolism. The approach works well if redundant pathways, cycles and branch points are present and if reaction steps that rearrange carbon chains are present, hence causing positional redistribution of isotope label. This principle therefore has been useful to resolve flux in central carbon metabolism, but less for other metabolic sub-networks of interest like sugar- or cell wall biosynthesis. Also, the steady state approach is not feasible under photoautotrophic 
conditions where $\mathrm{CO}_{2}$ is the sole carbon input [86]. In this case, methods based on isotope transient labeling are applicable. In isotopically non-stationary (INST) ${ }^{13} \mathrm{C}-\mathrm{MFA}$ analysis isotopically transient measurements are used to estimate flux by non-linear optimization $[87,88]$. Young et al. [89] first used the ${ }^{13} \mathrm{C}$-INST-MFA approach to predict photoautotrophic fluxes in a unicellular microalga. Feeding ${ }^{13} \mathrm{CO}_{2}$ to growing cultures of Synechocystis sp. PCC6803 they measured intracellular metabolite pool size and isotope labeling pattern by using GC-MS and LC-MS/MS. Photoautotrophic fluxes were estimated by an advanced INST-MFA algorithm [88]. They quantitatively estimated the fluxes of all Calvin-Benson-Bassham (CBB) cycle relations as well as photorespiratory fluxes and identified pathways responsible for reduce cyanobacterial productivity. Non-stationary isotope labeling approaches require acquisition of isotope labeling data in time series and resolving of intracellular fluxes in leafs by application of ${ }^{13} \mathrm{CO}_{2}$-pulse labeling to whole plants has proven to be a non-trivial task. A first study to measure fluxes of photoautotrophic metabolism in a plant leaf was reported by Szecowka et al. [90,91]. They estimated photosynthesis metabolic fluxes in Arabidopsis thaliana rosettes based on kinetic flux profiling (KFP) [90,92]. In difference to ${ }^{13} \mathrm{C}-$ INST-MFA, KFP does only use parts of the labeling information in the isotopomer labeling transient. As an important insight of this study, it was found that for some metabolites only parts of the total extracted metabolite pools appear to participate in the photosynthesis process. The presence of metabolically inactive pools must be accounted for in the modeling process to avoid distorted flux results. Ma et al. [93] first used INST-MFA to provide detailed flux maps of autotrophic leaf metabolism in Arabidopsis thaliana. After measuring approximately 1400 independent mass isotopomer states, INST-MFA was used to estimate 136 intracellular fluxes by least-squares parameter regression which was performed by publically available software package Isotopomer Network Compartmental Analysis (INCA) [94]. They compared flux states for low-light grown and high-light acclimated plants, labeled with ${ }^{13} \mathrm{CO}_{2}$ under low- and high-light conditions, respectively. The results correctly describe wellknown light-dependent differences in photosynthesis and carbon partitioning. For example, the photorespiration pathway increases under high light conditions and the carboxylation to oxygenation ratio of ribulose-1,5-bisphosphate carboxylase oxygenase was realistically estimated. This demonstrates that this ${ }^{13} \mathrm{C}$-INST-MFA based technique is adequate to probe photosynthesis.

Another application of flux analysis based on transient isotopic labeling in plants was reported for a non-photosynthetic system, heterotrophically grown Arabidopsis cell suspension cultures [95]. The authors studied the regulation of plant cell wall precursor metabolism by applying dynamic metabolic flux analysis (DMFA), based on fitting a kinetic model to transient labeling 
measurements. The kinetic rate constants of first order mass action rate equations were determined by fitting the model to the dynamic labeling and metabolite concentration measurements. Simulated carbon flow in the cell wall synthesis network showed that the sucrose invertase is mainly responsible for the entry of external carbon (sucrose) into metabolism. Due to the underlying kinetic model framework, Metabolic Control Analysis (MCA) could be performed to predict the key regulatory steps that affect carbon flow to cell wall synthesis. It was shown that phospho-glucomutase has a positive regulatory role and UDP-GlcA 4 epimerase has a negative regulatory role in carbon flow to cell wall synthesis.

\section{Conclusions and outlook}

We provided an overview on the important field of CB modeling in plants with focus on aspects of model reconstruction and simulation. Out of space limitation the overview might not mention all relevant work. For example, we did not include a number of relevant publications on unicellular photosynthetic alga. Along with others $[3,96]$ we argue that in order to be efficient for predictive metabolic engineering, plant genome-scale metabolic models must become more accurate and comprehensive. Besides the availability of a steadily increasing number of plant genomes and other sequencing information, development of high-throughput experimental determination of enzyme function might be important here. Also, since plants are complex multicellular organisms with elaborate life cycles, combination of CB models with other modeling formalisms $\left[18^{*}, 21^{*}\right]$ might point the way towards whole plant models.

\section{Acknowledgements}

JS gratefully acknowledges the Office of Basic Energy Sciences of the US Department of Energy for support of his laboratory's work on metabolic flux analysis (Office of Science, Office of Basic Energy Sciences, Chemical Sciences, Geosciences, and Biosciences Division, grant no. DEAC0298CH10886). Funding by the Laboratory Directed Research and Development 
program (LDRD) at Brookhaven National Laboratory to J.S. under contract with the U.S. Department of Energy is appreciated as well. 
Figure legends

Figure 1: Principle of exploring flux solution space in CB models. A) Structure of a toy network demonstrating redundancy by cell-internal parallel pathways. As commonly the case in real models, for most reaction bounds there are no reliable estimates available, in which case an arbitrarily large value (1000) is chosen to signify the "unboundedness". B) to G): Computational flux solutions. B) By Flux Balance Analysis (FBA) the rate through output $r_{8}$ was maximized, growing as large as allowable by the flux limitation imposed on the input $r_{1}$. The procedure found one possible internal flux distribution, which contains an unbounded / thermodynamically infeasible loop. (C, D) Unique and loopless solutions can be obtained if the exchanges across the system boundary are fixed and a secondary optimization is added to minimize the flux sum of internal reactions. Minimization of the sum of absolute flux values (C), or the sum of squared fluxes (D) are frequently being used. E) Flux Variability Analysis (FVA) delimits the entire solution space by the ranges of values the reactions can take. F) An extension of FVA, looplessFVA, eliminates loop solutions. G) Shrinking the solution space to a single solution by adding constraint equations derived from ${ }^{13} \mathrm{C}-\mathrm{MFA}$ is demonstrated $\left(r_{4} / r_{7}=2 ; r_{3} / r_{4}=1.5\right)$. All numerical outputs were generated with the COBRA toolbox [7] for MATLAB (version R2013a, http://www.mathworks.com) with TOMLAB CPLEX solver package (version 12.3, http://tomopt.com). 


\section{References and recommended reading}

Papers of particular interest, published within the annual period of review, have been highlighted as:

* of special interest

** of outstanding interest

1. Schwender J: Metabolic flux analysis as a tool in metabolic engineering of plants. Current Opinion in Biotechnology 2008, 19:131-137.

2. Lee JW, Na D, Park JM, Lee J, Choi S, Lee SY: Systems metabolic engineering of microorganisms for natural and non-natural chemicals. Nat Chem Biol 2012, 8:536-546.

3. Yoon JM, Zhao L, Shanks JV: Metabolic engineering with plants for a sustainable biobased economy. Annu Rev Chem Biomol Eng 2013, 4:211-237.

4. Lewis NE, Nagarajan H, Palsson BO: Constraining the metabolic genotype-phenotype relationship using a phylogeny of in silico methods. Nature reviews. Microbiology 2012, 10:291-305.

5. Edwards JS, Palsson BO: Systems properties of the Haemophilus influenzae Rd metabolic genotype. J Biol Chem 1999, 274:17410-17416.

6. Kim TY, Sohn SB, Kim YB, Kim WJ, Lee SY: Recent advances in reconstruction and applications of genome-scale metabolic models. Current opinion in biotechnology 2012, 23:617-623.

7. Schellenberger J, Que R, Fleming RM, Thiele I, Orth JD, Feist AM, Zielinski DC, Bordbar A, Lewis NE, Rahmanian S, et al.: Quantitative prediction of cellular metabolism with constraintbased models: the COBRA Toolbox v2.0. Nature protocols 2011, 6:1290-1307.

8. McCloskey D, Palsson BO, Feist AM: Basic and applied uses of genome-scale metabolic network reconstructions of Escherichia coli. Mol Syst Biol 2013, 9:661.

*9. Arnold A, Nikoloski Z: Bottom-up Metabolic Reconstruction of Arabidopsis and Its Application to Determining the Metabolic Costs of Enzyme Production. Plant Physiol 2014, 165:1380-1391.

Bottom-up reconstruction of an Arabidopsis core model of central metabolism, which, in difference to the genome-scale models is devoid of blocked reactions and dead-end metabolites. The authors demonstrated how to define a functional network of biochemical reactions without gap-filling algorithms. Although smaller than Arabidopsis genome-scale models, judged by the number of reactions that are functional for 
simulation of autotrophic biomass synthesis, simulations with the bottom-up reconstruction are comparable to the ones from genome-scale (top-down) reconstructions.

10. Arnold A, Sajitz-Hermstein M, Nikoloski Z: Effects of varying nitrogen sources on amino acid synthesis costs in Arabidopsis thaliana under different light and carbon-source conditions. PLoS One 2015, 10:e0116536.

*11. Borisjuk L, Neuberger T, Schwender J, Heinzel N, Sunderhaus S, Fuchs J, Hay JO, Tschiersch H, Braun HP, Denolf $P$, et al.: Seed Architecture Shapes Embryo Metabolism in Oilseed Rape. The Plant cell 2013, 25:1625-1640.

This study explores the metabolic heterogeneity within a plant organ, specifically cell layers of an oil accumulating seed. Physiological / biochemical measurements taken over the phase of seed storage accumulation reveal physiological gradients within the green photoheterotrophic storage tissue, which are coincident with light gradients, i.e. the shading effect of different layers of tissues around and within the seed. Using constraint-based modeling this metabolic heterogeneity is simulated by three differently parametrized sub-models and describes how the innermost layers grow essentially heterotrophic, while light levels received by outer layers are predicted to have substantial contribution of photosynthesis to the synthesis of storage compounds.

12. Cheung CY, Poolman MG, Fell DA, Ratcliffe RG, Sweetlove LJ: A Diel Flux Balance Model Captures Interactions between Light and Dark Metabolism during Day-Night Cycles in C3 and Crassulacean Acid Metabolism Leaves. Plant Physiol 2014, 165:917-929.

*13. Cheung CY, Williams TC, Poolman MG, Fell DA, Ratcliffe RG, Sweetlove LJ: A method for accounting for maintenance costs in flux balance analysis improves the prediction of plant cell metabolic phenotypes under stress conditions. The Plant journal 2013, 75:1050-1061.

This study is an assessment on how well flux states predicted by an Arabidopsis genome scale model compare to flux pattern resulting from experimental flux measurements by ${ }^{13} \mathrm{C}$-Metabolic Flux Analysis and to measured biomass yields in heterotrophically growing Arabidopsis cell cultures. While the metabolic cost of making cell biomass, in terms of energy cofactor requirements, are well accounted for in constraintbased models, there are significant energetic burdens related to cellular maintenance processes that typically have to be accounted for in the model as generic energy draining reactions. The authors show how attributing the cell maintenance cost to the consumption of both ATP and reductant improves the match to experimentally determined fluxes, irrespective of the chosen objective function.

14. de Oliveira Dal'Molin CG, Quek LE, Palfreyman RW, Brumbley SM, Nielsen LK: AraGEM, a genomescale reconstruction of the primary metabolic network in Arabidopsis. Plant Physiol 2010a, 152:579-589. 
*15. de Oliveira Dal'Molin CG, Quek LE, Palfreyman RW, Brumbley SM, Nielsen LK: C4GEM, a genomescale metabolic model to study C4 plant metabolism. Plant Physiology 2010b, 154:18711885.

In silico analysis of cell cooperation between mesophyll (M) and bundle sheath (BS) cells during photosynthesis in a C4 plant. The exchanges between the cell type models have to be balanced via common pools. Different subtypes of C4 photosynthesis are compared.

*16. Gomes de Oliveira Dal'Molin C, Quek LE, Saa PA, Nielsen LK: A multi-tissue genome-scale metabolic modeling framework for the analysis of whole plant systems. Front Plant Sci $2015,6: 4$.

This study presents a modeling framework for a whole plant model. Although at a multi-tissue scale, the model is still being consistent with the steady state principle inherent in Constraint-Based models. Metabolic models for leaf, stem and root are derived from the former published genome-scale reconstruction of Arabidopsis metabolism (AraGEM). The exchanges of the tissue models have to be balanced via common pools. In addition, a module for day-light metabolism has to be balanced with a nightmetabolism module. Daytime starch accumulation in leafs has to be equal to night time consumption. 17. Grafahrend-Belau E, Schreiber F, Koschützki D, Junker BH: Flux balance analysis of barley seeds: a computational approach to study systemic properties of central metabolism. Plant Physiology 2009, 149:585-598.

*18. Grafahrend-Belau E, Junker A, Eschenroder A, Muller J, Schreiber F, Junker BH: Multiscale metabolic modeling: dynamic flux balance analysis on a whole-plant scale. Plant physiology 2013, 163:637-647.

This study is an exploration of carbon partitioning in a whole-plant model. A multi-scale modeling approach (MMM) is used to simulate the changes in source-sink relations during the transition from early seedling development to the stage of seed filling. The model captures the dynamics of plant growth with timeresolves metabolite movements. At the whole plant level, a dynamic multiscale model simulates processes like gas exchange and primary photosynthesis at biophysical/physiological level. At the organ level (leaf, stem, seed), intracellular flux distributions are simulated by static FBA models. Deposition of fructan carbohydrate polymers in stem tissues is modeled as transitory carbon storage. The role of this transitory storage is being regarded as important for the overall yield of barley and similar grain crops. As a main outcome, the model realistically captures the transition of stem tissue from sink- to a source tissue during seed filling.

*19. Hay J, Shi H, Heinzel N, Hebbelmann I, Rolletschek H, Schwender J: Integration of a constraintbased metabolic model of Brassica napus developing seeds with $13 \mathrm{C}$-Metabolic Flux Analysis. Frontiers in Plant Science 2014, 5.

This study demonstrates integration of the Constrain Based approach of flux prediction (Flux Variability Analysis) with empirical flux data $\left({ }^{13} \mathrm{C}\right.$-metabolikc flux analysis), in order to reduce the solution space. In 
addition, the bottom-up approach of reconstruction of a plant metabolic network is reported. The model was developed alongside a carbon centric model used for ${ }^{13} \mathrm{C}$-MFA.

20. Lakshmanan M, Zhang Z, Mohanty B, Kwon JY, Choi HY, Nam HJ, Kim DI, Lee DY: Elucidating rice cell metabolism under flooding and drought stresses using flux-based modeling and analysis. Plant Physiol 2013, 162:2140-2150.

*21. Mallmann J, Heckmann D, Brautigam A, Lercher MJ, Weber AP, Westhoff P, Gowik U: The role of photorespiration during the evolution of $\mathrm{C} 4$ photosynthesis in the genus Flaveria. Elife 2014, 3:e02478.

This paper explores evolution of $\mathrm{C}_{4}$ photosynthesis by a combination of $\mathrm{CBM}$ and mechanistic models of photosynthesis. $\mathrm{C}_{4}$ photosynthesis is an adaptation to low atmospheric $\mathrm{CO}_{2}$. It involves distribution of photosynthetic $\mathrm{CO}_{2}$ fixation between two distinct cell types in leafs and establishes a $\mathrm{CO}_{2}$ concentration mechanism. In the study the authors combined a former published CB model of C4 photosynthesis (C4GEM, reference [16]) with a mechanistic model of C3-C4 intermediate photosynthesis. The mechanistic model was used to predict constraints for the stoichiometric model.

22. Melkus G, Rolletschek H, Fuchs J, Radchuk V, Grafahrend-Belau E, Sreenivasulu N, Rutten T, Weier D, Heinzel N, Schreiber F, et al.: Dynamic (1)(3)C/(1) H NMR imaging uncovers sugar allocation in the living seed. Plant Biotechnol J 2011, 9:1022-1037.

23. Mintz-Oron S, Meir S, Malitsky S, Ruppin E, Aharoni A, Shlomi T: Reconstruction of Arabidopsis metabolic network models accounting for subcellular compartmentalization and tissue-specificity. Proceedings of the National Academy of Sciences of the United States of America 2012, 109:339-344.

24. Nägele T, Weckwerth W: A workflow for mathematical modeling of subcellular metabolic pathways in leaf metabolism of Arabidopsis thaliana. Front Plant Sci 2013, 4:541.

25. Poolman MG, Miguet L, Sweetlove LJ, Fell DA: A genome-scale metabolic model of Arabidopsis and some of its properties. Plant Physiol 2009, 151:1570-1581.

*26. Poolman MG, Kundu S, Shaw R, Fell DA: Metabolic Trade-offs between Biomass Synthesis and Photosynthate Export at Different Light Intensities in a Genome-Scale Metabolic Model of Rice. Frontiers in Plant Science 2014, 5.

The authors explore the transition of a rice leaf from a phase of growth expansion where photosynthetic $\mathrm{CO}_{2}$ assimilation products serve biomass biosynthesis to the end of growth and export of photosynthate by a mature source leaf.

27. Poolman MG, Kundu S, Shaw R, Fell DA: Responses to light intensity in a genome-scale model of rice metabolism. Plant physiology 2013, 162:1060-1072. They study the adaptations of metabolism at different light intensities for an expanding leaf of rice. 
28. Saha R, Suthers PF, Maranas CD: Zea mays iRS1563: A Comprehensive Genome-Scale Metabolic Reconstruction of Maize Metabolism. PloS one 2011, 6:e21784.

*29. Schwender J, Hay JO: Predictive Modeling of Biomass Component Tradeoffs in Brassica napus Developing Oilseeds Based on in Silico Manipulation of Storage Metabolism. Plant physiology 2012, 160:1218-1236.

In this study changes in the flux distribution in central metabolism required to allow a change in biomass composition was predicted for an oil and protein storing seed. By changing the biosynthetic demands (biomass composition) a tradeoff between storage oil and protein was simulated. The approach makes network-wide predictions on flux variability range that must change in order to allow the simulated shift in biomass composition. Three distinct pathway usage patterns (metabolic phases) were detected. By flux sensitivities, reactions were categorized as oil- or protein responsive.

*30. Seaver SM, Bradbury LM, Frelin O, Zarecki R, Ruppin E, Hanson AD, Henry CS: Improved evidencebased genome-scale metabolic models for maize leaf, embryo, and endosperm. Front Plant Sci 2015, 6:142.

This study demonstrates integration of genome scale models with transcript data. The authors present revised Arabidopsis and maize genome-scale maize models. Tissue specific maize models were derived for the developing embryo and endosperm, based on an algorithm that achieves maximum agreement of presence or absence of reactions in the tissue specific network with high or low levels of expression, respectively. The tissue specific maize models are evaluated by obtaining a best fit of the models to flux data from respective studies reporting flux distribution based on ${ }^{13} \mathrm{C}$-metabolic flux analysis. In each case, an almost perfect fit shows that the tissue specific models are capable of assuming a flux distribution as found in the experimental flux analysis studies. Since the model was essentially fitted to flux data, this should not be mistaken as a proof of predictive power of the $C B$ model.

*31. Seaver SM, Gerdes S, Frelin O, Lerma-Ortiz C, Bradbury LM, Zallot R, Hasnain G, Niehaus TD, El Yacoubi $B$, Pasternak S, et al.: High-throughput comparison, functional annotation, and metabolic modeling of plant genomes using the PlantSEED resource. Proc Nat/ Acad Sci U S A 2014, $111: 9645-9650$.

PlantSEED is a metabolism-centric database to support gene annotation and metabolic model reconstruction in an integrated way. It was developed to streamline and automatize annotating plant genome sequences and reconstruction of metabolic models. Integrating across multiple genomes, the process can detect errors in gene annotations or predict new gene functions.

32. Simons M, Saha R, Amiour N, Kumar A, Guillard L, Clement G, Miquel M, Li Z, Mouille G, Lea PJ, et al.: Assessing the metabolic impact of nitrogen availability using a compartmentalized maize leaf genome-scale model. Plant Physiol 2014, 166:1659-1674. 
33. Töpfer N, Caldana C, Grimbs S, Willmitzer L, Fernie AR, Nikoloski Z: Integration of genome-scale modeling and transcript profiling reveals metabolic pathways underlying light and temperature acclimation in Arabidopsis. The Plant cell 2013, 25:1197-1211.

34. Töpfer N, Scossa F, Fernie A, Nikoloski Z: Variability of metabolite levels is linked to differential metabolic pathways in Arabidopsis's responses to abiotic stresses. PLOS Comput Biol 2014, 10:e1003656.

35. Michael TP, Jackson S: The First 50 Plant Genomes. Plant Genome 2013, 6.

36. Michael TP, VanBuren R: Progress, challenges and the future of crop genomes. Curr Opin Plant Biol 2015, 24:71-81.

37. Arabidopsis Genome I: Analysis of the genome sequence of the flowering plant Arabidopsis thaliana. Nature 2000, 408:796-815.

38. Janssen P, Goldovsky L, Kunin V, Darzentas N, Ouzounis CA: Genome coverage, literally speaking. The challenge of annotating 200 genomes with 4 million publications. EMBO Rep 2005, 6:397-399.

39. Orth JD, Conrad TM, Na J, Lerman JA, Nam H, Feist AM, Palsson BO: A comprehensive genomescale reconstruction of Escherichia coli metabolism--2011. Mol Syst Biol 201 1, 7:535.

40. Zhang P, Dreher K, Karthikeyan A, Chi A, Pujar A, Caspi R, Karp P, Kirkup V, Latendresse M, Lee C, et al.: Creation of a genome-wide metabolic pathway database for Populus trichocarpa using a new approach for reconstruction and curation of metabolic pathways for plants. Plant physiology 2010, 153:1479-1491.

41. Rhee SY, Mutwil M: Towards revealing the functions of all genes in plants. Trends Plant Sci 2014, 19:212-221.

42. Hanson AD, Pribat A, Waller JC, de Crecy-Lagard V: 'Unknown' proteins and 'orphan' enzymes: the missing half of the engineering parts list--and how to find it. Biochem J 2010 , 425:1-11.

43. Bradbury LM, Niehaus TD, Hanson AD: Comparative genomics approaches to understanding and manipulating plant metabolism. Curr Opin Biotechnol 2013, 24:278-284.

44. Chae L, Kim T, Nilo-Poyanco R, Rhee SY: Genomic signatures of specialized metabolism in plants. Science 2014, 344:510-513.

45. Yu X-H, Liu C-J: Development of an analytical method for genome-wide functional identification of plant acyl-coenzyme A-dependent acyltransferases. Analytical Biochemistry 2006, 358:146-148.

46. Gerdes S, El Yacoubi B, Bailly M, Blaby IK, Blaby-Haas CE, Jeanguenin L, Lara-Nunez A, Pribat A, Waller JC, Wilke A, et al.: Synergistic use of plant-prokaryote comparative genomics for functional annotations. BMC Genomics 2011, 12 Suppl 1:S2. 
47. Tanz SK, Castleden I, Hooper CM, Vacher M, Small I, Millar HA: SUBA3: a database for integrating experimentation and prediction to define the SUBcellular location of proteins in Arabidopsis. Nucleic acids research 2013, 41 :D1185-1191.

48. Hooper CM, Tanz SK, Castleden IR, Vacher MA, Small ID, Millar AH: SUBAcon: a consensus algorithm for unifying the subcellular localization data of the Arabidopsis proteome. Bioinformatics 2014, 30:3356-3364.

49. Khersonsky O, Tawfik DS: Enzyme promiscuity: a mechanistic and evolutionary perspective. Annu Rev Biochem 2010, 79:471-505.

50. Weng JK, Noel JP: The remarkable pliability and promiscuity of specialized metabolism. Cold Spring Harb Symp Quant Biol 2012, 77:309-320.

51. Weng JK: The evolutionary paths towards complexity: a metabolic perspective. New Phytol 2014, 201:1141-1149.

52. Nam H, Lewis NE, Lerman JA, Lee DH, Chang RL, Kim D, Palsson BO: Network context and selection in the evolution to enzyme specificity. Science 2012, 337:1101-1104.

53. Kunze R, Frommer WB, Flugge UI: Metabolic engineering of plants: the role of membrane transport. Metab Eng 2002, 4:57-66.

54. Yazaki K: Transporters of secondary metabolites. Curr Opin Plant Biol 2005, 8:301-307.

55. Linka N, Weber AP: Intracellular metabolite transporters in plants. Mol Plant 2010, 3:21-53.

56. Tohge T, Fernie AR: Lignin, mitochondrial family, and photorespiratory transporter classification as case studies in using co-expression, co-response, and protein locations to aid in identifying transport functions. Front Plant Sci 2014, 5:75.

57. Schwacke R, Schneider A, van der Graaff E, Fischer K, Catoni E, Desimone M, Frommer WB, Flugge Ul, Kunze R: ARAMEMNON, a novel database for Arabidopsis integral membrane proteins. Plant Physiol 2003, 131:16-26.

58. Thiele I, Palsson BO: A protocol for generating a high-quality genome-scale metabolic reconstruction. Nature protocols 2010, 5:93-121.

59. Weber, AP: Definition of plant metabolic networks. In Plant Metabolic Networks. Edited by Schwender J. Springer; 2009:9-38.

60. Weber AP, Linka N: Connecting the plastid: transporters of the plastid envelope and their role in linking plastidial with cytosolic metabolism. Annu Rev Plant Biol 201 1, 62:53-77.

61. Rolletschek H, Melkus G, Grafahrend-Belau E, Fuchs J, Heinzel N, Schreiber F, Jakob PM, Borisjuk L: Combined noninvasive imaging and modeling approaches reveal metabolic compartmentation in the barley endosperm. The Plant cell 2011, 23:3041-3054.

62. Hay JO, Schwender J: Metabolic network reconstruction and flux variability analysis of storage synthesis in developing oilseed rape (Brassica napus L.) embryos. Plant $J$ 2011a, 67:526-541. 
63. Hay JO, Schwender J: Computational analysis of storage synthesis in developing Brassica napus L. (oilseed rape) embryos: flux variability analysis in relation to ${ }^{13} \mathrm{C}$ metabolic flux analysis. Plant $J 2011 \mathrm{~b}, 67: 513-525$.

64. Pilalis E, Chatziioannou A, Thomasset B, Kolisis F: An in silico compartmentalized metabolic model of Brassica napus enables the systemic study of regulatory aspects of plant central metabolism. Biotechnol Bioeng 2011, 108:1673-1682.

65. Liu L, Shen F, Xin C, Wang Z: Multi-scale modeling of Arabidopsis thaliana response to different CO conditions: From gene expression to metabolic flux. J Integr Plant Biol 2015. doi: [10.1111/jipb.12370]

66. Schuetz R, Kuepfer L, Sauer U: Systematic evaluation of objective functions for predicting intracellular fluxes in Escherichia coli. Mol Syst Biol 2007, 3:119.

67. Schuetz R, Zamboni N, Zampieri M, Heinemann M, Sauer U: Multidimensional optimality of microbial metabolism. Science 2012, 336:601-604.

68. Colombie S, Nazaret C, Benard C, Biais B, Mengin V, Sole M, Fouillen L, Dieuaide-Noubhani M, Mazat JP, Beauvoit B, et al.: Modelling central metabolic fluxes by constraint-based optimization reveals metabolic reprogramming of developing Solanum lycopersicum (tomato) fruit. Plant J 2015, $81: 24-39$.

69. Bonarius HPJ, Schmid G, Tramper J: Flux analysis of underdetermined metabolic networks: The quest for the missing constraints. Trends in Biotechnology 1997, 15:308-314.

70. Reed JL: Shrinking the metabolic solution space using experimental datasets. PLoS computational biology 2012, 8:e1002662.

71. Mahadevan $\mathrm{R}$, Schilling $\mathrm{CH}$ : The effects of alternate optimal solutions in constraint-based genome-scale metabolic models. Metabolic Engineering 2003, 5:264-276.

72. Lewis NE, Hixson KK, Conrad TM, Lerman JA, Charusanti P, Polpitiya AD, Adkins JN, Schramm G, Purvine SO, Lopez-Ferrer D, et al.: Omic data from evolved E. coli are consistent with computed optimal growth from genome-scale models. Mol Syst Biol 2010, 6:390.

73. Machado D, Herrgard M: Systematic evaluation of methods for integration of transcriptomic data into constraint-based models of metabolism. PLoS Comput Biol 2014, 10:e1003580.

74. Kim MK, Lun DS: Methods for integration of transcriptomic data in genome-scale metabolic models. Comput Struct Biotechnol J 2014, 11:59-65.

75. Hoppe A: What mRNA Abundances Can Tell us about Metabolism. Metabolites 2012, 2:614631.

*76. Schwender J, König C, Klapperstück M, Heinzel N, Munz E, Hebbelmann I, Hay JO, Denolf P, Bodt SD, Redestig $\mathrm{H}$, et al.: Transcript abundance on its own cannot be used to infer fluxes in central metabolism. Frontiers in Plant Science 2014, 5. 
Using in-vitro cultured plant tissue (developing seed), it was tested if a genotypic difference in metabolic flux in central metabolism can be correlated with changes in the abundance of transcripts of relevant enzymes and metabolite transporters. With a few exceptions, differential activity in the major pathways (glycolysis, TCA cycle, amino acid, and fatty acid synthesis) was not reflected in contrasting abundances of the relevant transcripts.

77. King ZA, Lloyd CJ, Feist AM, Palsson BO: Next-generation genome-scale models for metabolic engineering. Current Opinion in Biotechnology 2015, 35:23-29.

78. Reznik E, Mehta P, Segre D: Flux imbalance analysis and the sensitivity of cellular growth to changes in metabolite pools. PLoS Comput Biol 2013, 9:e1003195.

79. Kim PJ, Lee DY, Kim TY, Lee KH, Jeong H, Lee SY, Park S: Metabolite essentiality elucidates robustness of Escherichia coli metabolism. Proc Natl Acad Sci U S A 2007, 104:1363813642.

80. Chung BK, Lee DY: Flux-sum analysis: a metabolite-centric approach for understanding the metabolic network. BMC Syst Biol 2009, 3:117.

81. Riemer SA, Rex R, Schomburg D: A metabolite-centric view on flux distributions in genomescale metabolic models. BMC Syst Biol 2013, 7:33.

82. Schwender J: Experimental flux measurements on a network scale. Frontiers in Plant Science $2011,2$.

83. O'Grady J, Schwender J, Shachar-Hill Y, Morgan JA: Metabolic cartography: experimental quantification of metabolic fluxes from isotopic labelling studies. Journal of experimental botany 2012, 63:2293-2308.

84. Li L, Hur M, Lee J-Y, Zhou W, Song Z, Ransom N, Demirkale C, Nettleton D, Westgate M, Arendsee Z, et al.: A systems biology approach toward understanding seed composition in soybean. BMC Genomics 2015, 16:S9.

*85. Schwender J, Hebbelmann I, Heinzel N, Hildebrandt T, Rogers A, Naik D, Klapperstück M, Braun HP, Schreiber F, Denolf P, et al.: Quantitative Multilevel Analysis of Central Metabolism in Developing Oilseeds of Oilseed Rape during in Vitro Culture. Plant Physiol 2015, 168:828-848.

In this study, variation in seed biomass composition was explored among several genotypes of Brassica napus by measurement of metabolite-, flux- and enzyme activity profiles in parallel. The results indicate that during seed development metabolic regulation in parts of central metabolism might be important for carbon partitioning between storage oil and starch.

86. Roscher A, Kruger NJ, Ratcliffe RG: Strategies for metabolic flux analysis in plants using isotope labelling. J Biotechnol 2000, 77:81-102.

87. Wiechert W, Noh K: From stationary to instationary metabolic flux analysis. Adv Biochem Eng Biotechnol 2005, 92:145-172. 
88. Young JD, Walther JL, Antoniewicz MR, Yoo H, Stephanopoulos G: An elementary metabolite unit (EMU) based method of isotopically nonstationary flux analysis. Biotechnol Bioeng 2008, 99:686-699.

89. Young JD, Shastri AA, Stephanopoulos G, Morgan JA: Mapping photoautotrophic metabolism with isotopically nonstationary (13)C flux analysis. Metabolic Engineering 2011, 13:656665.

90. Szecowka M, Heise R, Tohge T, Nunes-Nesi A, Vosloh D, Huege J, Feil R, Lunn J, Nikoloski Z, Stitt M, et al.: Metabolic fluxes in an illuminated Arabidopsis rosette. The Plant cell 2013, 25:694714.

91. Heise R, Arrivault S, Szecowka M, Tohge T, Nunes-Nesi A, Stitt M, Nikoloski Z, Fernie AR: Flux profiling of photosynthetic carbon metabolism in intact plants. Nat Protoc 2014, 9:1803-1824.

92. Yuan J, Bennett BD, Rabinowitz JD: Kinetic flux profiling for quantitation of cellular metabolic fluxes. Nat Protoc 2008, 3:1328-1340.

93. Ma F, Jazmin LJ, Young JD, Allen DK: Isotopically nonstationary $13 \mathrm{C}$ flux analysis of changes in Arabidopsis thaliana leaf metabolism due to high light acclimation. Proc Natl Acad Sci U S A 2014, $111: 16967-16972$.

94. Young JD: INCA: a computational platform for isotopically non-stationary metabolic flux analysis. Bioinformatics 2014, 30:1333-1335.

95. Chen X, Alonso AP, Shachar-Hill Y: Dynamic metabolic flux analysis of plant cell wall synthesis. Metabolic Engineering 2013, 18:78-85.

96. Collakova E, Yen JY, Senger RS: Are we ready for genome-scale modeling in plants? Plant Sci 2012, $191-192: 53-70$. 
Figure 1

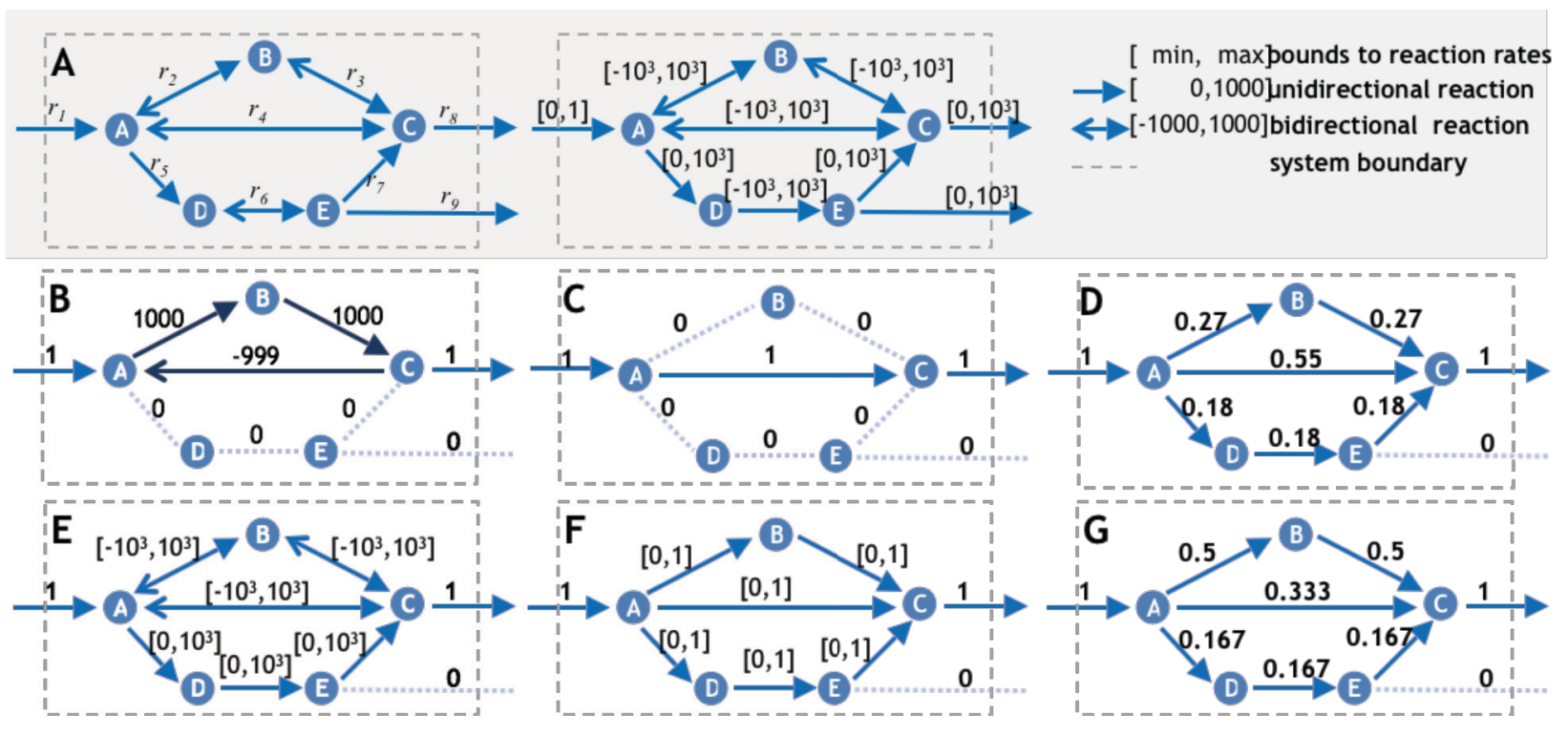


Graphical Abstract

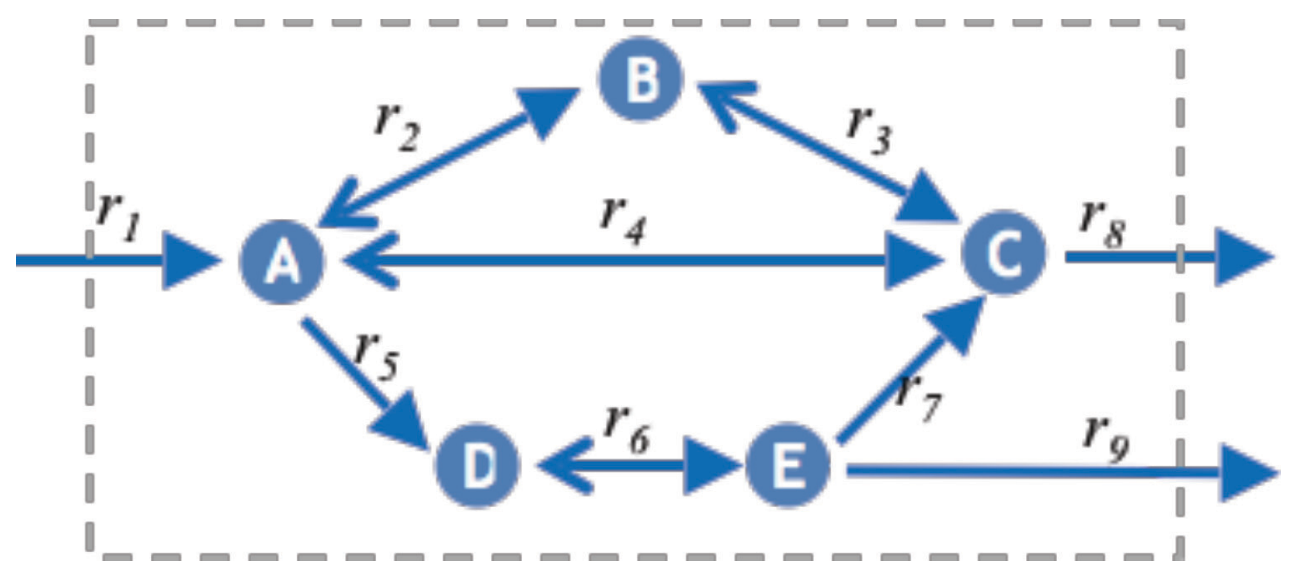

